\title{
Prevalence of human cytomegalovirus congenital INFECTION IN PORTUGUESE NEWBORNS
}

\author{
P Paixão (ppaixao.mic@fcm.unl.pt) ${ }^{1}, \mathrm{~S}$ Almeida², P Gouveia², L Vilarinho3 ${ }^{3}, \mathrm{R}$ Vaz Osório 3 \\ 1. Faculty of Medical Sciences, Lisbon, Portugal \\ 2. Hospital Centre Cova da Beira, Covilhã, Portugal \\ 3. Jacinto de Magalhães Institute of Medical Genetics, Oporto, Portugal
}

Human cytomegalovirus (HCMV) is considered the most frequent cause of congenital infection, occurring in 0.2 to $2.2 \%$ of all live births. Since this is a wide range of prevalences observed in different studies, it would be desirable to investigate the prevalence of this infection at national level. The aim of this study was the evaluation of the national prevalence of HCMV congenital infection. We analysed a total of 3,600 Guthrie cards collected from Portuguese newborns during a period of 14 months (August 2003 to September 2004). The cards covered all regions of Portugal and were proportional to the number of births in each region. A heat DNA extraction method was used, followed by DNA amplification by nested PCR. Sensitivity and specificity of this method were evaluated as $93 \%$ and $100 \%$, respectively, using 28 cards from HCMV-positive and 280 cards from HCMV-negative children. The national prevalence of congenital HCMV was determined as $1.05 \%$ (95\% confidence interval: $0.748-1.446$ ). This is the first study of the prevalence of HCMV congenital infection at national level in Portugal. It suggests that Portugal may have one of the highest prevalences of congenital HCMV infection in Europe.

\section{Introduction}

Human cytomegalovirus (HCMV) is considered the most frequent cause of congenital infection, occurring in 0.2 to $2.2 \%$ of all live births [1]. Since this is a wide range of prevalences observed in different studies, it would be desirable to study the prevalence of this infection at national level. To our knowledge, only one study about the prevalence of HCMV congenital infection in Portugal has been published. It used urine detection by the shell-vial method, and the estimated prevalence was $0.7 \%$ [2]. However, the above study was performed at only one hospital, making it difficult to extrapolate the results to the national prevalence. For an estimation of the national prevalence, urine samples from all the regions in the country would have to be collected, proportional to the number of births from each region, and HCMV would have to be detected by cell culture. However, this approach is not feasible to perform.

Dried blood spots (DBS), also known as Guthrie cards, have been used both for late detection of HCMV congenital infection (after the first three weeks of life) and for epidemiological studies with promising results $[3,4]$. Since all the DBS collected in Portugal for metabolic neonatal screening are sent to a national reference laboratory - the Jacinto de Magalhães Institute of Medical Genetics - and stored there for several years, the use of these cards allow sampling from all Portuguese regions, which would be very difficult with viruria tests.

The aim of this work was the study of the prevalence of HCMV congenital infection in Portuguese newborns, based on the above collection of DBS. The methodology used was adapted from a technique previously described by an Italian team [5]. The first step of this study was therefore the determination of the sensitivity and specificity of this technique, when compared with the reference method, i.e. the detection of HCMV in urine by cell culture. The second step was the study of the national prevalence.

\section{Material and methods}

\section{Sensitivity and specificity of nested PCR on DBS}

Samples

Sensitivity was studied using Guthrie cards from 28 children with HCMV congenital infection as determined by detection of HCMV in the urine by shell-vial culture during the first three weeks of life. Specificity was studied using Guthrie cards from 280 neonates without HCMV infection (no detection of HCMV in the urine by shell-vial culture during the first three weeks of life). All cards were from children between one month and eight years of age at the time of our study. The DBS had been collected in the first week of their life and stored at the Jacinto de Magalhães Institute of Medical Genetics. For our study, this institute sent the cards, with the parents' consent, to the Hospital Centre Cova da Beira.

\section{Molecular analysis}

We used a heat-induced DNA extraction method, followed by amplification of HCMV DNA in a nested polymerase chain reaction (PCR), using a protocol adapted from Barbi et al. (2000) [5]. Each sample was tested in triplicate.

Blood was eluted from the Guthrie cards, and the DNA was extracted using a heat-induced protocol. A nested PCR protocol was used to amplify a region of the HCMV genome coding for the gp58 subunit of glycoprotein B [6]. The following oligonucleotide primers were used:

Outer primers:

- gB 1: 5'-gAggACAACgAAATCCTgTTgggCA-3'

- gB2: 5'-gTCgACggTggAgATACT-gCTgAgg-3'

Inner primers:

- gB3: 5'ACCACCgCACTgAggAATgTCAg-3'

- gB4: 5'TCAATCATgCgTTTgAAgAggTA-3' 
The sensitivity of this nested-PCR technique had previously been tested by us and shown to consistently detect HCMV in a suspension of 900 copies/ml of the laboratory strain AD-169 (unpublished data). Two different concentrations of AD-169 were processed as positive controls in each set of PCRs, and water was processed as negative control in quadruplicate. Disks punched from blank Guthrie cards were processed as additional negative controls and tested along with the samples. The cards that gave a positive result in at least one of the triplicate amplifications were retested with a new series of disks.

\section{Viral culture}

Viral cultures were grown at the Hospital Centre Cova da Beira or at the Hospital de Santa Cruz, using shell-vial assays in human foetal lung fibroblast cells (MRC-5 line), using a protocol previously described by protocol Gleaves et al. (1985) [7], with minor modifications. The cells were analysed by immunofluorescence using anti-HCMV pool I.E.A.+E.A. monoclonal antibodies which give a typical nuclear signal in HCMV infected cells.

Prevalence of HCMV congenital infection in Portuguese newborns

We studied a total of 3,600 dried blood spots (DBS), that had been collected from Portuguese newborns during a period of 14 months (August 2003 to September 2004) and sent to the national screening laboratory. These newborns were from all regions of Portugal, including the Azores and Madeira. The number of Guthrie cards studied was proportional to the number of births in each region (data from the Jacinto Magalhães Institute of Medical Genetics). Within each region, the cards were randomly chosen and sent anonymously to the Hospital Centre Cova da Beira. The study was approved by the Comissão Nacional de Protecção de Dados (National Data Protection Commission).

DNA extraction and PCR were performed as described above.

\section{Results}

Sensitivity, specificity and negative and positive predictive values of the nested-PCR on DBS

\section{Specificity}

Of the 280 cards from uninfected individuals, 267 were negative in all three PCR tests. The remaining 13 cards were positive in one of the three PCR tests. On repetition, these 13 cards were negative in all three PCR tests (total: 1/6 positive tests). Therefore, no card among the 280 negative controls had more than one positive amplification out of six, and this was established as the cut-off for discrimination between positive and negative cards. The 13 single-positive PCR results were assumed to have been caused by a laboratory contamination during the amplification step, and were considered false-positive results.

\section{Sensitivity}

Of the 28 cards from HCMV-infected individuals, 26 had more than three positive amplifications in six PCR tests and were considered positive. Two cards were under the cut-off described above ( $\leq 1 / 6$ positive amplifications) and were considered negative.

With these results, the sensitivity, specificity, negative and positive predictive values of this nested-PCR with the criteria described above were, respectively, 93\%, 100\%, 99\% and 100\%.

Prevalence of HCMV congenital infection in Portuguese newborns

The above method and cut-off were used to estimate the prevalence of HCMV congenital infection in Portugal. Of the 3,600 Guthrie cards tested, 38 were positive, according to the criteria described above. This corresponds to a prevalence of $1.05 \%$ (95\% confidence interval; exact binomial method: 0.748-1.446).

\section{Discussion}

The importance of studying the prevalence of congenital HCMV infection, the most frequent congenital infection [1], should not be underestimated. Updated evaluations of the impact of this infection are needed in order to raise awareness of the true burden of congenital HCMV infection and disease, allocate public health resources, and determine the cost-effectiveness or cost-benefit of potential interventions [8]. Determination of the congenital HCMV prevalence in each country would certainly benefit this purpose.

This is the first study on the prevalence of HCMV congenital infection at national level in Portugal. The 3,600 cards tested covered all regions of Portugal and were proportional to the number of births in each region, so that the samples represented all the Portuguese territory. To our knowledge, this is also the first study using Guthrie cards from all regions in one country to estimate a national prevalence, although one multicentric study used this technology for the determination of the prevalence of congenital HCMV in Italy [4].

The methodology used in the present study was adapted from a method described by an Italian team [5], which was reported to have $100 \%$ sensitivity and $99 \%$ specificity compared to the reference method, virus isolation in cell culture. Therefore, the first step of our study was to determine the sensitivity and the specificity of the adapted protocol used by us.

For the sensitivity analysis, we included DBS from all children diagnosed with CMV congenital infection between 1995 and 2001 who had been tested with the reference method at the Hospital Centre Cova da Beira and the Hospital de Santa Cruz and who fulfilled the inclusion criteria, i.e. signed informed consent from the parents and availability of a DBS collected in the first week of life before receiving any blood transfusion. A total of 28 cards were tested, obtained from symptomatic and asymptomatic infections, but also from children for whom clinical information was not available, which was the case for the two negative results. These two had had a positive urine culture result and we therefore consider them false-negatives.

Because the viral load was not determined in the above mentioned laboratories in 2004 and urine specimens were not preserved until 2007 (when they introduced routine determination of CMV viral load), the relationship between false-negative results, clinical information and low viral loads could not be ascertained in this study.

Interestingly, our recent experience with an external proficiency panel of samples (CMV DBS, organised by Quality Control for Molecular Diagnostics from the European Society of Clinical Virology) suggests that low viral loads could be the main factor responsible for false-negative results (unpublished data). Other possible explanations for the two false-negative results could be ineffective DNA extraction or the presence of inhibitory substances in the specimen; this was not checked in this evaluation because the technique described by Barbi et al. (2000) does not include an internal control [5]. Nevertheless, the $100 \%$ sensitivity obtained by the Italian team suggests that PCR inhibition is not a significant problem inherent to this technique. 
Our results of $93 \%$ sensitivity and $100 \%$ specificity were encouraging and allowed us to proceed with the aim of this study, the determination of the prevalence of HCMV congenital infection in Portuguese newborns. The observed prevalence of $1.05 \%$ was within the expected range [1], albeit a little higher than in some of the latest European reports $[4,9,10]$. According to a recent metaanalysis of selected studies which had used the reference method and analysed at least 800 urine or saliva samples, the prevalence in European countries ranged from 0.3 to $0.5 \%$, but only studies from Belgium, Denmark, Italy, Sweden, and the United Kingdom were included in this review [11].

One possible explanation for our results could be the high seroprevalence of around 80\% in pregnant women in Portugal [12], because a positive correlation between maternal seroprevalence and birth prevalence has been described: one meta-analysis suggested maternal seroprevalence as a significant predictor of birth prevalence, with every $10 \%$ increase in maternal seroprevalence corresponding to a $0.26 \%$ increase in birth prevalence [8]. However, this cannot be the only explanation, since Barbi et al. (2006) also described a seroprevalence of $80 \%$ in women of childbearing age, whereas it identified only $0.18 \%$ of newborns with congenital infection in. Interestingly, the same team reported $0.47 \%$ congenital infection in a previous study [13]. Whether this discrepancy was the result of different methodologies or sample size $(9,032$ pooled DBS in the study from 2006 versus 1,268 urine cultures in 1998) or due to other factors, is unclear.

In the present study, a higher sampling could have narrowed the confidence interval, but for practical reasons, the sample size had to be limited to 3,600 cards. Since the methodology implied that each card must be tested in triplicate, followed by a further three amplifications for those with a positive result, more than 10,000 individual nested-PCR reactions had to be performed in the current setup of the study.

Considering that the sensitivity of the method was 93\%, the maximum proportion of expected false-negative results would not have had a significant influence on the final prevalence $(1.05 \%$ could have been $1.14 \%$ ). On the other hand, the specificity in the first phase of the study was $100 \%$, assuring a very low probability of false-positive tests in the second phase. However, specificity was studied in only 280 cards and it cannot be ruled out that a falsepositive result may have occurred if more cards had been analysed.

Since the cards for prevalence determination were sent anonymously, we could not obtain clinical information, including maternal serological evolution during pregnancy Another study coordinated by the Portuguese Society of Paediatrics, currently addresses this point in order to figure out the relative importance of maternal primary and recurrent infections in the Portuguese children with congenital CMV infection.

With around 105,000 births per year, a prevalence of $1.05 \%$ translates to about 1,103 children (between 785 and 1,518, with a $95 \%$ confidence interval of $0.748-1.446$ ) born in Portugal each year with this congenital infection. Assuming that around $11 \%$ of these infections will be symptomatic at birth [8], about 121 (between 86 and 167) of the infected newborns will have signs and/ or symptoms, and at least half of them will present late sequelae. To these numbers must be added an estimated $13.5 \%$, i.e. about 149 (between 106 and 205) children with asymptomatic infection at birth, who will suffer from late sequelae, particularly sensorineural hearing loss and cognitive impairment [11].
In conclusion, for the first time in Portugal a nationwide study using DBS allows us to estimate the number of children congenitally infected with CMV. Our data suggest that Portugal may have one of the highest prevalences of congenital CMV infection in Europe, although nationwide studies in other European countries are needed before any conclusions can be drawn.

\section{Aknowledgements}

We thank Prof Maria Barbi for critical review of the manuscript. This work was supported by grants from the FEDER (programa Saúde XXI, Portuguese Ministry of Health, 2003).

\section{References}

1. Revello MG, Gerna G. Pathogenesis and prenatal diagnosis of human cytomegalovirus infection. J Clin Virol. 2004;29(2):71-83.

2. Lopo S, Vinagre E, Palminha P. Vírus Citomegalo. Avaliação do programa nacional de vacinação. $2^{0}$ Inquérito serológico nacional - Portugal Continental, 2001 2002. [Cytomegalovirus. Assessment of the national vaccination programme. 2nd national serological survey - continental Portugal, 2001-2002]. Lisbon, Portugal: Direcção Geral de Segurança; $2004 a$.

3. Barbi M, Binda S, Primache V, Luraschi C, Corbetta C. Diagnosis of congenital Cytomegalovirus Infection by detection of viral DNA in dried spots. Clin Diagn Virol. 1996;6(1):27-32.

4. Barbi M, Binda S, Caroppo S, Calvario A, Germinario C, Bozzi A, et al, Multicity Italian study of congenital cytomegalovirus infection. Pediatr Infect Dis J. 2006;25(2):156-9.

5. Barbi M, Binda S, Primache V, Caroppo S, Dido P, Guidotti P et al. Cytomegalovirus DNA detection in Guthrie cards: a powerful tool for diagnosing congenital infection. J Clin Virol. 2000;17(3):159-65.

6. Wakefield A, Fox J, Sawyerr A, Taylor J, Sweenie C, Smith M, et al, Detection of Herpesvirus DNA in the Large Intestine of Patients with Ulcerative Colitis and Crohn's Disease Using the Nested Polymerase Chain Reaction. J Med Virol. 1992;38(3):183-90.

7. Gleaves C, Smith T, Shuster E, Pearson G. Comparison of Standard Tube and Shell Vial Cell Culture Techniques for the Detection of Cytomegalovirus in Clinical Specimens. J Clin Microbiol. 1985;21(2):217-21.

8. Kenneson A, Cannon M. Review and meta-analysis of the epidemiology of congenital cytomegalovirus (CMV) infection. Rev Med Virol. 2007;17(4):253-76.

9. Naessens A, Casteels A, Decatte L, Foulon W. A serologic strategy for detecting neonates at risk for congenital cytomegalovirus infection. J Pediatr. 2005;146(2):194-7.

10. Schlesinger $Y$, Reich D, Eidelman AI, Schimmel MS, Hassanin J, Miron D. Congenital cytomegalovirus infection in Israel: screening in different subpopulations. Isr Med Assoc J. 2005;7(4):237-40.

11. Dollard S, Grosse S, Ross D. New estimates of the prevalence of neurological and sensory sequelae and mortality associated with congenital cytomegalovirus infection. Rev Med Virol. 2007;17(5):355-63.

12. Lopo S, Palminha P, Pité M, Caçador T, Vinagre E, Pereira MA, et al. Infecção Congénita pelo vírus citomegálico -Resultados preliminares de estudo prospectivo. [Congenital infection with cytomegalovirus - preliminary results of a prospective study]. Revista Portuguesa de Doenças Infecciosas 2004b;Aug-Dec:14-7.

13. Barbi M, Binda S, Primache V, Clerici D. Congenital cytomegalovirus infection in a northern Italian region. NEOCMV Group. Eur J Epidemiol. 1998;14(8):791-6.

This article was published on 5 March 2009.

Citation style for this article: Paixão P, Almeida S, Gouveia P, Vilarinho L, Vaz Osório R. Prevalence of human cytomegalovirus congenital infection in Portuguese newborns. Euro Surveill. 2009;14(9):pii=19135. Available online: http://www.eurosurveillance.org/ ViewArticle.aspx?ArticleId $=19135$ 